\title{
Comparative Analysis of Impulse and Impedance Tests to Detect Short Circuits within the W7-X Magnets
}

\author{
H. Ehmler, H. Fillunger, J. Baldzuhn, R. K. Maix, A. Jeckle, S. Parodi, T. Rummel, K. Riße and H. \\ Scheller
}

\begin{abstract}
The magnet system of the Wendelstein 7-X stellarator (W7-X) consists of 50 non-planar and 20 planar coils. In particular, the winding packs of the non-planar coils are densely packed and compressed in the area of inter-layer joints and coil terminations, which brings some risk of inter-turn and inter-layer short circuits.

The paper describes methods deducted from impulse and ac tests performed on the coils, which enable demonstrating the absence of short circuits and weak points of low inter-layer insulation resistance. In the impulse test, frequency and damping are compared for coils with and without short circuits and coils with an external low resistance bypass. With ac voltage, a drastically reduced impedance clearly indicates a short circuit. Therefore both tests represent excellent QA (quality assurance) tools for checking the uniformity of the electromagnetic performance of a serial production of magnet coils.
\end{abstract}

Index Terms - Electric variables measurement, Impedance measurement, Coils, Impulse testing, Short circuit currents.

\section{INTRODUCTION}

$\mathrm{M}$ agnetic field coils needed for fusion devices require high current densities. Therefore the conductors are densely compacted in the winding pack. The coil cases are constructed of high strength stainless steel materials to withstand the high electromagnetic forces. In all cases where conductors contain superconductive material like $\mathrm{Nb}$ - $\mathrm{Ti}$ composites, the coils are mounted in cryostats allowing operation at temperatures below $6 \mathrm{~K}$. As a consequence, the coils are hardly accessible once they are assembled. Therefore it is indispensable to check the quality of the coils during manufacture and before assembly with the best technology available.

Manuscript received September 20, 2005

H. Ehmler, J. Baldzuhn, K. Riße and T. Rummel are with the Max-PlanckInstitut für Plasmaphysik (IPP), Wendelsteinstr. 1, 17489 Greifswald, Germany (corresponding author Hartmut Ehmler, phone +49 (0)3834/882525, fax +49 (0)3834/88-2709, e-mail: Ehmler@ipp.mpg.de).

H. Fillunger and R. Maix are with the Technical University Wien, Atominstitut, Austria.

A. Jeckle is with ABB Service AG, Augsburg, Germany.

S. Parodi is with ASG, Ansaldo Superconduttori, Genova, Italy

H. Scheller is with BNN, Babcock Noell Nuclear, Würzburg, Germany.
The paper focuses on the W7-X non-planar coils [1-3]. A nonplanar coil consists of 108 turns which are wound in six double layers (DLLs). The DLLs are connected in series by five inter-layer joints. Each joint is contacted with quenchdetection cables, which enable electrical measurements of the individual DLL. The winding pack ground insulation consists of epoxy-resin reinforced glass fibers and is covered with conductive paint. The coil is embedded in a stainless steel casing which is electrically connected with the conductive paint (ground potential).

During the manufacture of the W7-X non-planar coils some critical areas in the vicinity of the inter-layer joints and coil terminals turned out to create short circuits. These were caused mainly by an inappropriate molding form during vacuum impregnation. By thoroughly checking the electrical variables throughout the manufacturing steps, it is possible to ensure a high quality level of the winding packs and finally of the completed coils. These variables are the resistance of the winding, the coil impedance at several frequencies and low voltage (impedance spectrum test), the resistance of the interturn insulation at moderate voltage (ac test and impulse test) and the resistance of the ground insulation at high dc voltage (HV-dc test). An inter-turn voltage is applied either by a strong ac power supply (ac test) or by a charged capacitance (impulse test) connected to the coil terminals. The ac test is carried out on winding packs and completed coils with $\leq 2$ $\mathrm{kV}_{\mathrm{p}}$ at $1-2 \mathrm{kHz}$. It is repeated at liquid He temperature during the coil tests at Saclay [4], before and after quench. The impulse test is done only on winding packs using a capacitance discharge of $20 \mathrm{nF}$ at $2 \mathrm{kV}_{\mathrm{p}}$. This voltage corresponds to an inter-turn voltage of $18.5 \mathrm{~V}$ and a voltage of $333 \mathrm{~V}$ across each DLL.

An inter-layer short circuit could be detected by any of the mentioned tests, whereas e.g. an inter-turn short circuit cannot be detected by a resistance measurement of the winding. This paper focuses on impedance spectrum test and impulse test.

\section{IMPEDANCE SPECTRUM TEST}

\section{A. Winding Pack}

Measurements were performed at frequencies of $50 \mathrm{~Hz}$ to 30 $\mathrm{kHz}$ using an RLC-meter at amplitude of $5 \mathrm{~V}$. Magnitude and 
phase angle of the measured impedance are compared with calculated results from an equivalent circuit. We modeled the impedance of a winding pack by a parallel resonance circuit, where a capacitance is in parallel to an inductance in series with a resistance, where the impedance is given by [5]

$Z=\frac{U}{I}=\frac{R+j \omega\left[L\left(1-\omega^{2} L C\right)-C R^{2}\right]}{\left(1-\omega^{2} L C\right)^{2}+(\omega C R)^{2}}$.

$R=0.125 \Omega$ is the resistance of the winding, $L=50 \mathrm{mH}$ the self inductance and $C=5 \mathrm{nF}$ the capacitance. These values can be used for all non-planar coils in good approximation, since the five different non-planar coil types have very similar electric variables. The inductance was calculated by the EFFIcode $[6,7]$, the resistance was measured and the capacitance was left as a free parameter to match the observed resonance frequency. Fig. 1a shows absolute value and phase angle of the impedance from a winding pack without conductive paint. Below $2 \mathrm{kHz}$ the winding pack behaves like an inductive load. At $10 \mathrm{kHz}$ the impedance has a sharp resonance. Above the resonance frequency it behaves like a capacitance. Measurements and calculation match very well.

\section{B. Completed Coil}

An inter-turn short circuit as well as the coil casing or even the conductive paint represent single secondary windings of a transformer; with the winding pack acting as the primary winding. The impedance is modified by the eddy currents flowing in the secondary winding. The resistance in (1) is modified into [8]:

$$
R=R_{1}+\frac{\omega^{2} M^{2} R_{2}}{\omega^{2} L_{2}^{2}+R_{2}^{2}} .
$$

In case of a coil with casing, $R_{1}$ denotes the resistance of the winding pack and $R_{2} \approx 120 \mu \Omega$ the resistance of the casing. $L_{2}=3.23 \mu \mathrm{H}$ is the self inductance of the casing and $M=0.36$ $\mathrm{mH}$ the mutual inductance between winding and casing. The inductances were calculated by the EFFI code and the casing resistance was measured by a $\mu \Omega$-meter (Schütz Meßtechnik MR 300C-A).

The inductance in (1) is modified into [8]:

$L=L_{1}-\frac{\omega^{2} M^{2} L_{2}}{\omega^{2} L_{2}^{2}+R_{2}^{2}}$,

where $L_{1}$ denotes the self inductance of the winding. For the casing, the skin effect is taken into account by an effective penetration depth. This depth is smaller than the average casing thickness of $\sim 6 \mathrm{~cm}$ for frequencies above $100 \mathrm{~Hz}$ which leads to an increase of the effective casing resistance proportional to $\sqrt{f}$. Fig.1b shows the impedance and phase angle of the calculation in comparison to the data. These measurements were performed with a spectrum analyzer (Hewlett Packard 4194a) in very fine frequency steps. Data and model show clearly that the inductive part of the impedance is strongly reduced and that the resonance frequency is shifted to a higher value around $20 \mathrm{kHz}$. The observed resonance is broader and the phase angle is smaller as in the calculation. This implies that not all losses were incorporated. Ac losses of the cable itself e.g. are not included in the calculation. Further deviations of model and measurements were found in the fine structure of the spectrum. Preliminary results from a refined model using a network analyzing code with the individual DLLs as discrete elements show a similar fine structure [9].

\section{Winding Pack with Inter-turn Short Circuit}

Fig. 1c shows the impedance spectrum of a winding pack as in Fig. 1a but with an inter-turn short circuit (AAB49). The measured resonance is much broader. For modeling, Eq. (1)(3) were used, but with $R_{2}$ as a free parameter. Since the contact resistance of the short circuit is unknown, this treatment seems justified for a first approach. A value of $R_{2}=$ $0.1 \Omega$ was found to match the measurements best. However, the model curves depend strongly on the input parameters, especially on the inductances. Therefore, the model should be refined.

\section{Coil with Inter-layer Short Circuit}

An artificial short circuit of individual DLLs was introduced via the quench detection cables on an embedded coil. Impedance spectra are shown in Fig. 2. Depending on the short circuited DLL, the reduction in impedance at $2 \mathrm{kHz}$ can be as large as a factor of three. The resonance shifts to a higher frequency and the phase angle (not shown) decreases by up to $25^{\circ}$ for frequencies below the resonance. No modeling of this case, e.g. by a network analyzing code, has been done so far.

With the impedance spectrum test, even "soft" short circuits of some hundred Ohms could be detected. Such "soft" short circuits were introduced by resistors connected in parallel to one DLL. The magnitude of $Z$ is reduced e.g. by $50 \%$ in the vicinity of the resonance, when a resistance of $100 \Omega$ is connected in parallel to DLL5.

\section{IMPULSE TEST}

The impulse test on winding packs of type 1 and 5 was performed with a Baker FP 212E device at ABB Service AG, Augsburg. The test turned out to be very sensitive to variations of most electric variables. By comparing the shape of the damped voltage curve, any disturbance or deviation from the design can be clearly detected. Disturbances may originate from variations in the conductor length, relative position of the conductors to each other, existence of surface treatment like conductive paint, inter-layer and inter-turn short circuits (or low insulation resistance) or even surrounding magnetic materials. Fig. 3 shows the impulse response of a winding pack with and without an artificial short circuit. One turn was short circuited by steel probes. In comparison to an intact winding pack, damping and frequency of the oscillation are increased by the short circuit. This reflects the same phenomena as in the impulse spectrum test (Fig. 1c), where resonance frequency and real part of the impedance are 
increased. The higher real power leads to stronger damping.

All impulse test responses of correctly produced winding packs fit into a narrow bandwidth. Fig. 4 shows the amplitude damping curves of different winding packs. Deviations clearly indicate short circuits or missing manufacturing steps (i.e. lack of conductive paint). Three winding packs (AAB16 already embedded in casing, AAB20, AAB51) had an inter-layer short circuit $(\sim 1 \mathrm{~m} \Omega)$ of DLL 5 in the conductor termination area. All short circuits were repaired. Fig. 5 shows the amplitude damping curve of AAB16. The curve after repair matches the curve from an intact coil. Fig. 5 also includes measurements with a low inter-layer insulation resistance. This resistance was artificially introduced via the quench detection cables, as for the impedance spectrum test. A resistance of up to some hundred Ohms can be detected by this method, as for the impedance spectrum test. It should be pointed out that a resistance of such magnitude does not affect the total winding resistance in a measurable way. The same holds for the impedance measured during the high voltage ac test at $2 \mathrm{kHz}$. Nevertheless such a defect would produce additional heating of the winding pack during a fast safety discharge of the magnet with the risk of coil damage.

\section{CONCLUSION}

The importance of electrical tests accompanying each production step was underlined. Early detection of a defect is possible. Some redundancy in tests (e.g. ac and impulse test) reduces the risk to overlook a failure. Exact margins for the expected values of electrical variables should be defined from the beginning and, if necessary, adapted during production according to the statistical scatter of measurements. Deviations must be treated within a mutually agreed nonconformity procedure where both parties, contractor and customer, work in a close collaboration for resolving technical and/or QA problems.

\section{REFERENCES}

[1] K. Risse et al., "Fabrication of the superconducting coils for Wendelstein 7-X“, Fusion Engineering and Design 66-68 (2003) p. 965969

[2] C. Sborchia, J. Baldzuhn, K. Risse, T. Rummel, H. Viebke: "Progress in the Design, Manufacture and Testing of the Wendelstein 7-X Superconducting Magnets", this conference WEM20R6

[3] K. Risse et al., "Design Changes and Impact on the Production of the Non-Planar Coils for the W7-X Experiment", this conference THM10R5

[4] J. Baldzuhn et al., "Recent Results of the Cold Tests performed on the Stellarator W7-X Coils" this conference MOA11PO09

[5] Zinske and Brunswig, "Lehrbuch der Hochfrequenztechnik", Springer 1965, p. 12.

[6] E. Harmeyer, IPP Greifswald, Germany, data available via ftp://ftp.ipp.mpg.de/pub/ipp/ebh/w7xcoils or on request from authors

[7] S. J. Sackett, User's Manual: "EFFI - A code for Calculating the Electromagnetic Field, Force, and Inductance in Coil Systems of Arbitrary Geometry, 1977, Lawrence Livermore Laboratory, California, UCID-17621

[8] Meinke and Gundlach, "Taschenbuch der Hochfrequenztechnik", Springer 1968, 3nd Edition, p. 30 eq. 12.6 corrected from $\mathrm{M}_{\text {into }} \mathrm{M}^{2}$, p. 31 eq. 12.11 denominator corrected from $\mathrm{L}_{2}$ into $\mathrm{L}_{2}{ }^{2}$.

[9] M. Köppen, IPP Greifswald, Germany, private communication.
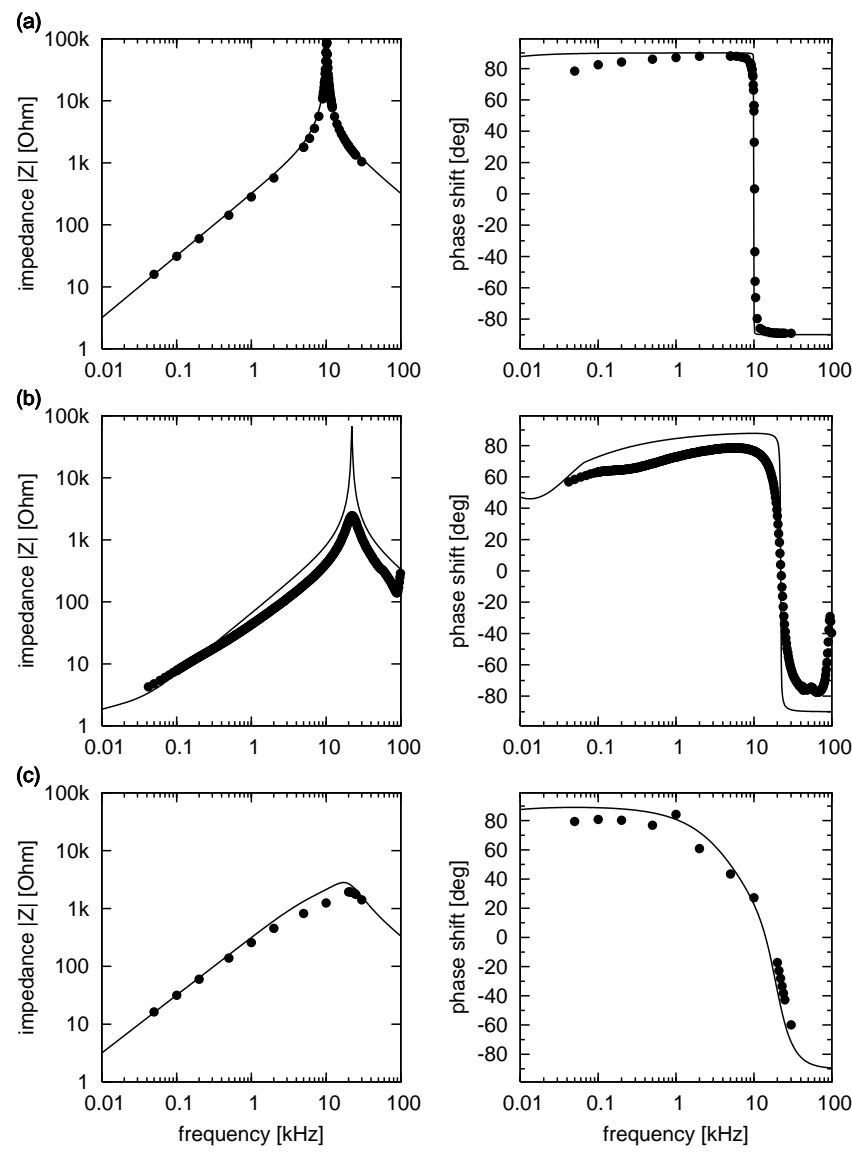

Fig. 1. Impedance spectra of winding packs and coils. Measured data (dots) are compared to calculations (curves). Left column shows magnitude and right column phase angle of impedance. (a) winding pack without conductive paint (AAB33), (b) embedded coil (AAB24), (c) winding pack without conductive paint and inter-turn shortcut (AAB49).

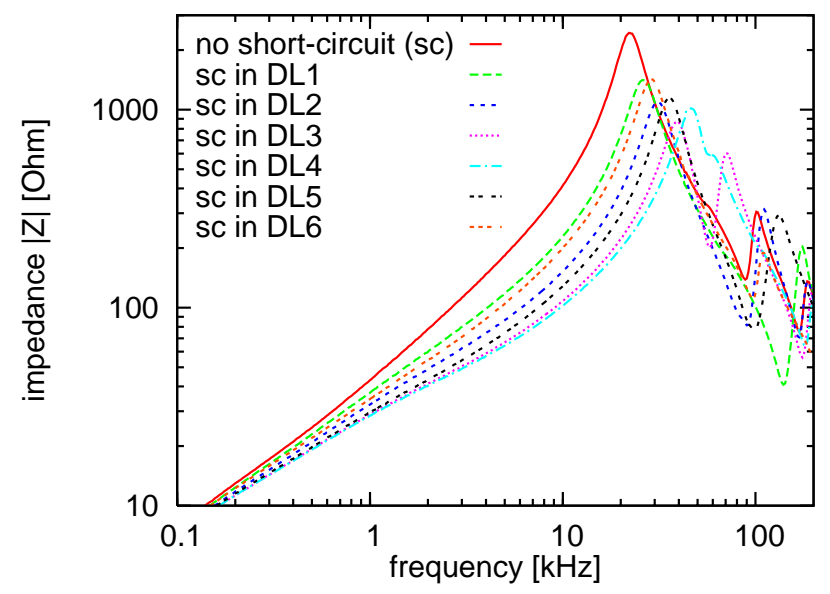

Fig. 2. Impedance spectrum of coil AAB24. An artificial shortcut of different double layers was introduced by the quench detection cables. The figure shows one spectrum without short circuit and six spectra with a short circuit in one of the six double layers. 


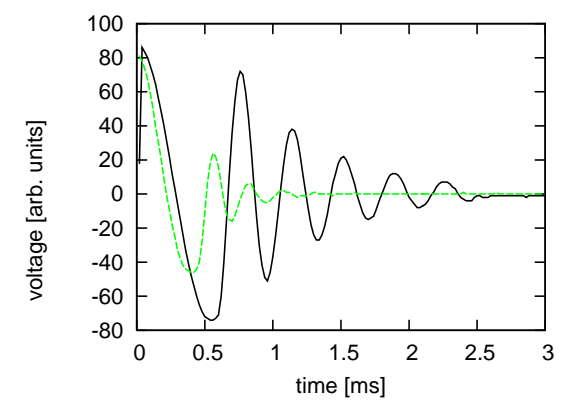

Fig. 3. Impulse response of winding pack AAB40 with (dashed curve) and without an artificial short circuit (solid curve). One turn was short circuited by steel probes. In comparison to an intact winding pack, damping and frequency of the oscillation are increased by the short circuit.

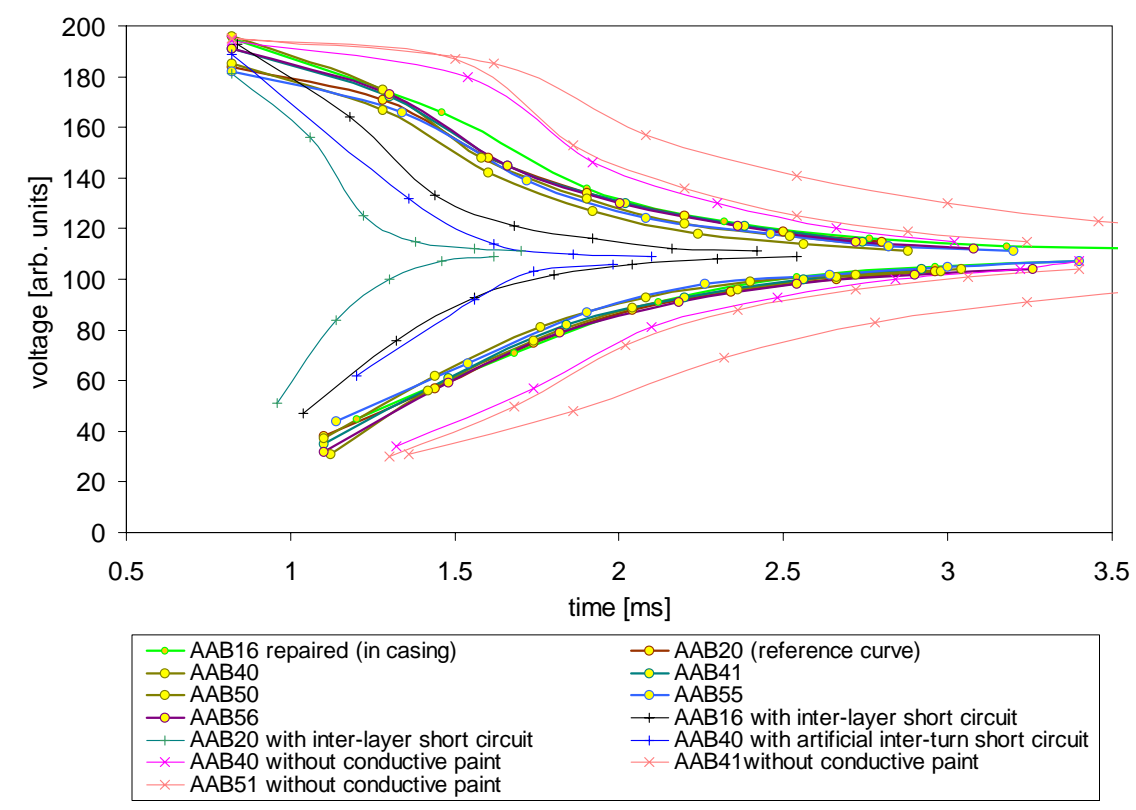

Fig. 4. Impulse test results (amplitude damping curves) of different winding packs. All curves of correctly produced winding packs fit into a narrow bandwidth (AAB40, AAB41, AAB50, AABAAB55, AAB56). Deviations clearly indicate short circuits (AAB16, AAB40) or missing manufacturing steps i.e. lack of conductive paint (AAB41, AAB51).

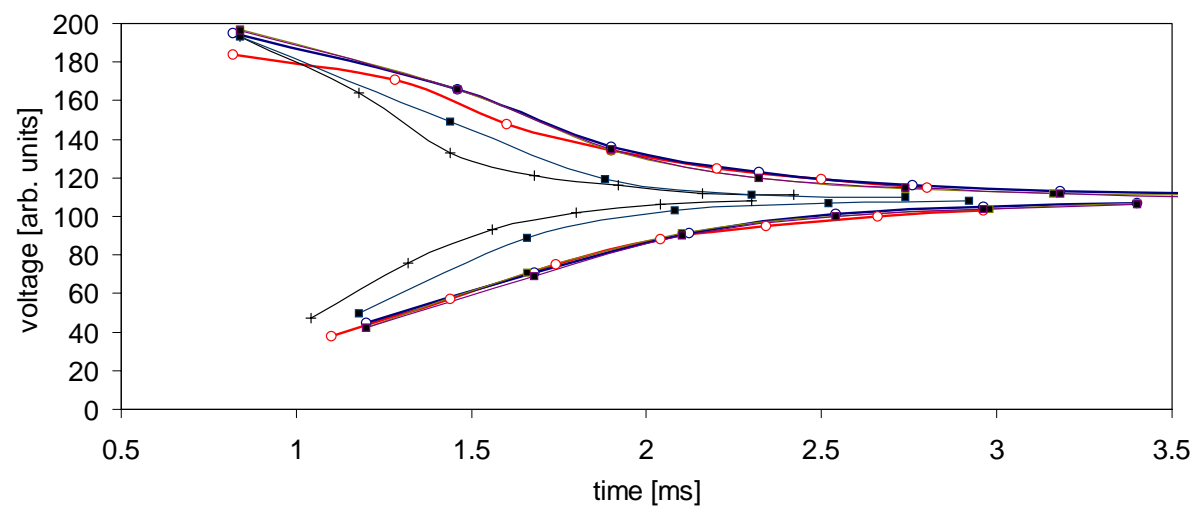

\begin{tabular}{ll|}
\hline- - AAB20 (reference curve) & - AAB16 repaired (in casing) \\
- AAB16 with inter-layer short circuit & - -ABB16, inter-layer short circuit of 100 Ohm \\
- - AAB16, inter-layer short circuit of $1 \mathrm{kOhm}$ & - AAB16 inter-layer short circuit of $2.5 \mathrm{kOhm}$ \\
\hline
\end{tabular}

Fig. 5. Amplitude damping in impulse test of an embedded coil which had an inter-layer short circuit (AAB16). This short circuit was repaired. The curve after repair matches the curve from an intact coil. Also included are measurements with a low inter-layer insulation resistance. This resistance was artificially introduced via the quench detection cables. A low resistance of less than some hundred Ohms can be detected by this method. 